\title{
Clinical aspects of the acquired immune deficiency syndrome in the United Kingdom
}

\author{
J N WEBER,* D J CARMICHAEL, + N SAWYER, † A J PINCHING, \& AND \\ J R W HARRIS*
}

From the *Praed Street Clinic and the Departments of $†$ Medicine and $\neq$ Immunology, St Mary’s Hospital, London W2

SUMMARY Between August and November 1983, seven new patients with AIDS were seen at this hospital; two with disseminated Kaposi's sarcoma, and five with opportunist infections, of whom three have died. We present the case histories of the five patients with opportunist infections, which show the wide clinical range of AIDS and suggest that the pattern of infection in the UK may differ from that reported in the USA.

\section{Introduction}

The acquired immune deficiency syndrome (AIDS) is an unprecedented epidemic of immunocompromise, resulting in opportunist infections, Kaposi's Sarcoma, and other unusual neoplasms. ${ }^{1-9}$ It was first documented in mid-1981, and over 3000 cases had been reported by December 1983, including 36 in the United Kingdom. ${ }^{10}{ }^{11}$ Over $70 \%$ of cases of AIDS have occurred in homosexual men. ${ }^{12}$ The epidemiological features are compatible with a sexually transmitted, blood borne agent. ${ }^{13}$ The clinical features suggest a variable latent period, followed by prodromal non-specific symptoms of weight loss, fever, night sweats, and malaise. ${ }^{34}$ The most common opportunist infection is Pneumocystis carinii pneumonia, which occurs in $50 \%$ of all cases of AIDS in the United States of America. ${ }^{12}$

\section{Case reports}

\section{CASE 1}

A 22 year old male homosexual prostitute was well until June 1982, when he presented to his general practitioner with fever, night sweats, and weight loss. A chest $x$ ray showed an opacity in the right third interspace, and he was admitted to a local hospital for investigation. On admission, he was noted to have a swinging fever, oral candidiasis, ulcerating perianal herpes simplex, and pancytopenia. The

Address for reprints: Dr J N Weber, Jefferiss Research Wing, The Praed Street Clinic, St Mary's Hospital, London W2 1NY

Accepted for publication 10 February 1984 opacity seen on chest $x$ ray proved to be a bifid rib. Blood cultures were repeatedly sterile, and a bone marrow examination showed only normocellular marrow. A therapeutic trial of broad spectrum antibiotics failed to control the fever, and treatment with oxymethalone was started for the pancytopenia. The patient was discharged, and he returned to his home town. The fever, oral candidiasis, and pancytopenia persisted. Between January and June 1983 he was admitted to another hospital on seven occasions for investigation. Oesophageal candidiasis was confirmed by endoscopy, and two episodes of chest infection resolved on treatment with cotrimoxazole without an agent being identified. Over this period, seven grand mal epileptic fits were reported, although there was no history of epilepsy. A computed tomography scan of the head and an electroencephalogram showed no focal abnormality, and examination of the cerebrospinal fluid showed no cells, but a raised protein concentration $(1 \cdot 6 \mathrm{~g} / 1)$. The patient became increasingly lethargic and apathetic and was transferred to this hospital in August 1983.

On admission, the patient was appreciably wasted (weight $38.5 \mathrm{~kg}$ ) with oral candidiasis, ulcerating infection with herpes simplex perianally and in the anal canal, and with tender hepatomegaly. He was anaemic, with a blood haemoglobin concentration of $1.03 \mathrm{~g} / \mathrm{dl}$ and a total lymphocyte count of $0.6 \times$ $10^{9} / \mathrm{l}$. There was anergy to three recall antigens, and an absolute depletion of $\mathrm{T}$ helper lymphocyte phenotype $\left(0 \cdot 23 \times 10^{9} / 1\right)$. Gastroduodenoscopy showed candidal oesophagitis, with a $2 \mathrm{~cm}$ duodenal ulcer. Despite frequent small haemoptyses, the chest $x$ ray remained clear. Lung function tests, however, showed a pronounced reduction in carbon monoxide 
transfer factor (TICO) (30\% of that predicted), with preserved spirometry. A fibreoptic brochoscopy with lavage and transbronchial biopsy showed no pathogens.

The herpetic ulceration and fever settled on treatment with oral acyclovir $1 \mathrm{~g}$ a day, and both returned on withdrawal of this drug, which has since been maintained at $200 \mathrm{mg}$ three times a day. The candidiasis is controlled, but not eradicated, by amphotericin lozenges, nystatin suspension, and oral ketoconazole. Despite symptomatic healing of his duodenal ulcer the wasting has been resistant to treatment; however, there has recently been a $4 \mathrm{~kg}$ weight gain. His mental state improved spontaneously, and his fits have been controlled with anticonvulsant treatment.

The patient has never travelled outside the UK and had no known contact with AIDS, although clearly his previous occupation put him at risk of contact with a large number of casual partners. $\mathrm{He}$ is currently living independently as an outpatient.

\section{CASE 2}

A 52 year old male homosexual executive was well until March 1983, when he developed anorexia, severe weight loss $(>12 \mathrm{~kg})$, and overwhelming malaise. A pancreatic neoplasm was suspected, but whole body computed tomography and endoscopic retrograde cholangiopancreatography gave negative results in August, although the latter procedure showed pronounced oral and oesophageal candidiasis. In view of the probable diagnosis and severe constitutional symptoms, treatment with prednisolone $40 \mathrm{mg}$ a day was started and the patient went to Malta to convalesce. One week later he developed fever, night sweats, and confusion. On his return to the UK, blood cultures grew Salmonella typhimurium, which was also isolated from the stool, and the appropriate antibiotics were started. The septicaemia persisted, and the patient was transferred to this hospital.

On admission he was noted to have florid oral candidiasis and a fluctuating mental state. There was lymphopenia (lymphocyte count $0.8 \times 10^{9} / 1$, T helpers $\left.0.18 \times 10^{\circ} / \mathrm{l}\right)$ with otherwise unremarkable blood tests. Blood culture confirmed infection with Salmonella typhimurium, with extensive colonisation of the gut and skin. Computed tomography scanning of the head, electroencepalogram, and examination of cerebrospinal fluid failed to find a specific cause for the altered mental state. A polyomavirus, which was neither JC nor BK, was later isolated from the urine. The relevance of this virus is uncertain at present. The antibiotic regimen was augmented to include all three drugs to which the organism was sensitive (gentamicin, mecillinam, and colistin), but it was never possible to eradicate the resistant salmonella bacteraemia. He died of toxaemia after nine weeks of continuous septicaemia.

The patient had visited the USA and Canada on numerous occasions over the preceding five years, with casual sexual contacts. There was no known contact with AIDS. Eighteen months before admission, he had experienced a febrile illness with pronounced lymphocytosis, atypical mononuclear cells, and negative Paul-Bunnell test reactions. This was thought at the time to have been glandular fever not caused by Epstein-Barr virus or cytomegalovirus.

\section{CASE 3}

A 28 year old male homosexual air steward was well until June 1983 when he developed sudden onset of diarrhoea while in Australia. Stool culture grew Salmonella ohio; oral candidiasis was also noted. On his return to the UK, his diarrhoea persisted, and his general practitioner treated him with ampicillin. A follow up stool examination by his employers showed persistence of the salmonella, but his symptoms settled; he had lost five $\mathrm{kg}$ in weight, but this was constant thereafter.

In August, his diarrhoea returned, and he was noted to have oral candidiasis; in addition, he was lymphopenic (lymphocyte count $0.8 \times 10^{9} / 1$ ). He was referred to the Praed St Clinic, where he was found to have anergy to three recall antigens, and to have $T$ helper depletion $\left(0.23 \times 10^{9} / 1\right)$; his chest $x$ ray was clear. He was observed as an outpatient until September, when he complained of a sudden onset of dyspnoea over three days. He was admitted immediately, and his chest $x$ ray showed bilateral mid-zone infiltrates, compatible with early atypical pneumonia. He was cyanosed and hypoxic, with an arterial oxygen partial pressure of $7.7 \mathrm{kPa}$ $(58 \mathrm{~mm} \mathrm{Hg})$. There had been no sputum production. Transtracheal aspiration was performed immediately, but showed no pathogens. Treatment with high dose co-trimoxazole was started $(1.92 \mathrm{~g} 12$ hourly $)$ because of the risk of Pneumocystis carinit pneumonia. A fibreoptic bronchoscopy was performed 24 hours later with lavage and transbronchial biopsy. Candida albicans was seen free and within type II pneumocytes, and a diagnosis of probable candidal pneumonia was made. Treatment with amphotericin and miconazole was started, and erythromycin was added because of the possibility of infection with legionella.

Despite the appropriate antibiotic regimen, the patient's condition worsened clinically, and the hypoxia deepened. Serial chest $x$ rays showed a rapid progression of the pulmonary infiltrates, causing generalised bilateral opacity by the fifth day. On day 6 he was moved to the intensive treatment unit for 
ventilation, but had an asystolic cardiac arrest after a short period of intubation and ventilation; prolonged resuscitation was unsuccessful.

The patient had visited the USA regularly over the preceding five years, and had had many casual sexual contacts in cities throughout the world. There was no known contact with AIDS.

\section{CASE 4}

A 34 year old male homosexual air steward was well until August 1983, when he developed sudden onset of diarrhoea. He attended his local STD clinic, where Entamoeba histolytica was isolated from the stool. He was treated with metronidazole $400 \mathrm{mg}$ twice daily for one week, and the diarrhoea abated. One week after the completion of treatment, the diarrhoea returned. The stool was watery, offensive, and pale in colour and contained globules of fat. The diarrhoea was episodic, lasting two or three days, and the episodes were associated with fever and night sweats. Weight loss of $5 \mathrm{~kg}$ was noticed over the next month. A routine examination at work showed oral candidiasis, and a full blood count showed lymphopenia (lymphocyte count $0.8 \times 10^{9} / \mathrm{l}$ ). He was referred to the STD clinic at this hospital.

On examination he was noted to be slightly wasted, with mild oral candidiasis, and without lymphadenopathy; the abdomen was normal to palpation. Repeated stool culture showed Entamoeba histolytica (zymodeme 111) and a variety of nonpathogenic amoebae. Sigmoidoscopy showed normal mucosa, and a rectal biopsy showed areas of chronic inflammation, with no evidence of amoebic infiltration. Barium enema, isotope liver scan, and abdominal ultrasound gave negative results. Studies of cellular immunity showed cutaneous anergy to three recall antigens and a reduction in $\mathrm{T}$ helper phenotypes $\left(0 \cdot 22 \times 10^{9} / 1\right)$. Upper gastrointestinal intubation and biopsy were performed at the Central Middlesex Hospital, and oocysts of Cryptosporidium spp were seen on the jejunal mucosa and were later isolated from the stool by a concentration technique.

The patient is currently being evaluated for treatment of cryptosporidium, which can also cause symptomatic diarrhoea in immunocompetent homosexual patients. ${ }^{14} 15$ He has had numerous visits to the USA and elsewhere, and has had many sexual partners in the USA.

\section{CASE 5}

A 47 year old homosexual man was well until October 1983, when he noted severe malaise and a persistent sore throat. He presented to the Praed St Clinic, where a routine examination showed slight lymphopenia (lymphocyte count $1.3 \times 10^{9} / 1$ ). He declined to return for follow up, and one month later began to develop a dry cough, night sweats, and shortness of breath on exertion. His general practitioner referred him to the chest clinic at this hospital, where a chest $x$ ray showed bilateral mid-zone pulmonary infiltrates, consistent with an atypical pneumonia.

On admission in November, cyanosis, florid oral candidiasis, and tachypnoea were noted. The spleen was palpable $3 \mathrm{~cm}$ below the costal margin. Blood gases on air showed marked hypoxia. The lymphocyte count was $0.8 \times 10^{9} / 1$ (T helpers $0.14 \times$ $\left.10^{9} / \mathrm{l}\right)$. A fibreoptic bronchoscopy with lavage and transbronchial biopsy was performed immediately, but no organisms were positively identified. Treatment was started with co-trimoxazole $1.92 \mathrm{~g} 12$ hourly for presumptive Pneumocystis carinii pneumonia, and parenteral antifungal treatment with amphotericin was started 48 hours later. The chest $x$ ray appearances continued to worsen, and the blood gases deteriorated over the next four days; he was transferred to the intensive treatment unit. A diffuse maculopapular skin rash developed eight days after starting co-trimoxazole. This drug reaction is commonly seen in the context of AIDS. ${ }^{16}$ The antibiotic regimen was augmented to cover legionella with erythromycin, and the patient was ventilated when unable to maintain his arterial partial oxygen pressure. He died of pneumonia of unknown cause on the 14th day.

The patient had visited Canada in 1978, but had not been to the USA or to Haiti in the past five years. He had had large numbers of casual sexual partners in the UK, but had no known contacts with AIDS.

\section{Discussion}

Owing to the different environment, the pattern of infectious diseases in the USA differs from that in the UK. For this reason, the pattern of opportunistic infections in AIDS in this country cannot be assumed to be identical to that previously reported from the USA. Analysis of the small number of cases at this hospital indicates some notable differences from the general American pattern.

Pneumocystis carinii was not isolated in any of these cases, despite adequate transbronchial biopsy and bronchial lavage with appropriate staining. Open lung biopsy was not possible in the two fatal cases of pneumonia, owing to the rapid development of severe hypoxia; however, neither of these patients responded to empirical treatment with high dose cotrimoxazole. The cause of the fulminant pneumonia was not identified in one case (case 5) despite postmortem lung biopsy. In case 3 , a probable diagnosis of Candida albicans pneumonia was made on cytological examination only. It would be 
expected that Pneumocystis spp would be identified by fibreoptic bronchoscopy in $85 \%$ of cases. ${ }^{22}$

The incidence of infection with Salmonella spp (cases 2 and 3) shows a noticeable variance from that in the USA, although it is similar to European reports of cases from Africa. ${ }^{17}$ Salmonella species are not currently listed as being associated with AIDS in the epidemiological protocol of the Centers for Disease Control. ${ }^{18}$ In case 2 Salmonella typhimurium was the principal pathogen, and AIDS was confirmed by the additional oesophageal candidiasis. In case 3 the salmonella was restricted to the gut, and blood cultures gave consistently negative results. It is reasonable to surmise that Salmonella spp may be of greater clinical importance in AIDS in the UK. As a facultative intracellular pathogen it is an appropriate secondary infection to this type of immune deficit.

Cytomegalovirus (CMV) was not excreted in the blood, stool, or urine in any of these patients, in contrast to the common finding of CMV viruria in the USA. ${ }^{19}$ In a study of asymptomatic homosexual men in San Francisco, CMV viuria was found in $32 \%$ of seropositive men, whereas a comparative study of 100 asymptomatic homosexual men at this hospital showed that only $1 \%$ of seropositive men excreted $\mathrm{CMV}$ in the urine.$^{20} \mathrm{CMV}$ was not cultured from any site in all the above cases.

Candida albicans was found in all the patients with AIDS at this hospital, both as oral candidiasis with large easily visible plaques on the buccal mucosae, and as oesophageal candidiasis at endoscopy or by radiology. The oesophageal candida was not associated with overt ulceration in these cases. The aetiology of the duodenal ulcer in case 1 was not established, but no opportunist agent was found in the ulcer crater. The presence of oral candidiasis was an early sign in all these cases, and was found before the isolation of other opportunist infections.

In cases 3 and 5 , a mild prodromal illness of less than eight weeks' duration without any significant weight loss was reported. This preceded fulminant pneumonia, which developed rapidly. There was a history of only three days of dyspnoea before admission to hospital in both these cases. This rapid development has been rare amongst the reported cases in the USA, and precluded open lung biopsy. ${ }^{3-5}$ Lymphopenia, with a total lymphocyte count in peripheral blood of less than $1.5 \times 10^{9} 1$, preceded overt opportunist infection in every case. In case 5 lymphopenia (lymphocyte count $1.3 \times 10^{9}$ l) was the only laboratory abnormality six weeks before the onset of fatal pneumonia. By comparison, in case 1 lymphopenia has been present continuously for 18 months with oesophageal candidiasis and perianal herpes simplex but without life threatening infection.

In conclusion, we recommend that asymptomatic homosexual men should have a complete physical $\stackrel{\mathbb{D}}{\vec{D}}$ examination, with special attention to the buccal mucosa for oral candidiasis and lesions of Kaposi's ? sarcoma; the entire skin area should be examined. A $\overrightarrow{\vec{F}}$ full blood count with differential and platelet count should be taken at every visit, and a serum sample stored at $-80^{\circ} \mathrm{C}$ for future reference. A throat swab for candida should be taken. If facilities exist for the investigation of high risk patients, estimation of total $T$ helper numbers and in vivo testing of cellular immune response with intradermal recall antigens (purified protein derivative, candida, and streptokinase or streptokinase-streptodornase) may be of value. Immunological abnormalities may occur in healthy, asymptomatic homosexual men. ${ }^{2021}$ The finding of lymphopenia, oral candidiasis (in the absence of recent antibiotic treatment), or both, is important and warrants further investigation for occult opportunist infection.

The investigation of homosexual men with symptoms of severe malaise, loss of weight, night sweats, fevers, or prolonged diarrhoea should be undertaken urgently. In addition to the tests above, strenuous efforts should be made to investigate the gastrointestinal tract for pathogens, the chest should be $x$ rayed, and lung function tests should be performed. The combination of symptoms with lymphopenia, oral candidiasis, or both is an indication for urgent admission to hospital and extensive investigation.

We thank the referring physicians, Drs Dawson, Green, and Chanarin; the physicans and staff of the respiratory laboratory; Dr I Trotman of the department of gastroenterology at Central Middlesex Hospital; the staff of the department of microbiology, particularly Drs D Jeffries and A Maddocks; Dr D Coleman and the department of cytology; and most importantly Sister M Anthony and the nursing staff of Almroth Wright Ward, St Mary's Hospital. The management of patients with AIDS in hospital requires a multidisciplinary approach, and it is not possible to thank by name all the physicians and technicians who have been concerned with the diagnosis and treatment of these patients.

\section{References}

1. Centers for Disease Control. Pneumocystis pneumonia - Los Angeles. $M M W R$ 1981; 30:250-2.

2. Centers for Disease Control. Kaposi's sarcoma and pneumocystis pneumonia among homosexual men-New York City and California. $M M W R$ 1981; 30:305-8.

3. Gottleib MS, Schroff R, Schanker HM, et al. Pneumocystis pneumonia and mucosal candidiasis in previously healthy homosexual men: evidence of a newly acquired immunodeficiency. N Engl J Med 1981; 305: 1425-31.

4. Siegal FP, Lopez P, Hammer GS, et al. Severe acquired immune deficiency in male homosexuals, manifested by chronic perianal herpes simplex lesions. $N$ Engl J Med 1981;
305: 1439-44. . 
5. Masur H, Michelis MA, Greene JB, et al. An outbreak of community acquired Pneumocystis carinii pneumonia: initial manifestation of a cellular immune dysfunction. N Engl J Med 1981;305: 1431-8.

6. Centers for Disease Control. Diffuse, undifferentiated nonHodgkins lymphoma in homosexual men-USA. MMWR 1982;31:277-9.

7. Ziegler JL, Drew WL, Miner RC, et al. Outbreak of Burkitt's-like lymphoma in homosexual men. Lancet 1982; ii: 631-3.

8. Miller JR, Barrett RE, Britton CB, et al. Progressive multifocal leukoencephalopathy in a male homosexual with T-cell immune deficiency. $N$ Engl $J$ Med 1982;307:1436-8.

9. Centers for Disease Control. Cryptosporidiosis-assessment of chemotherapy in males with acquired immuno-deficiency syndrome (AIDS). MMWR 1982;31:589-92.

10. Centers for Disease Control. Weekly statistics on AIDS, December 1983. MMWR, 1983;32:389-91.

11. Anonymous. Surveillance of AIDS. Br Med J 1984; 288: 576

12. Jaffe HW, Bregman DJ, Selik RM. Acquired immune deficiency syndrome in the USA; the first 1000 cases. $J$ Infect Dis 1983; 148:339-45.

13. Francis DP, Curran JW, Essex M. Epidemic acquired immune deficiency syndrome: epidemiologic evidence for a transmissable agent. JNCI 1983;71:1-4.
14. Tzipori S. Cryptosporidiosis in animals and humans. Microbiol Rev 1983; 47:84-96.

15. Weber JN, Philip S. Human cryptosporidiosis. N Engl J Med 1983;309: 1326 .

16. Jaffe HW, Abrams DI, Ammann AJ, Lewis BJ, Golden JA. Complications of co-trimoxazole in the treatment of AIDS associated pneumocystis carinni pneumonia in homosexual men. Lancet 1983; ii:1109-11.

17. Clumeck N, Mascart-Lemone F, de Maubeuge J, Brenes D, Marcelis L. AIDS in black Africans. Lancet 1983; i:642.

18. Anonymous. The case definition of AIDS used by the CDC for epidemiologic surveillance. Atlanta: Centers for Disease Control, 15 March 1983; circular: 3pp

19. Mintz L, Drew LW, Miner RC, Braff E H. Cytomegalovirus infections in homosexual men. Ann Intern Med 1983;99:326-9.

20. Pinching AJ, McManus TJ, Jeffries DJ, et al. Studies in cellular immunity in male homosexuals in London. Lancet 1983;ii: 126-30.

21. Pinching AJ, Weber JN, Rogers L, et al. One year follow-up of immunological abnormalities in homosexual men in London. Ann NY Acad Sci (in press).

22. Coleman DL, Dodek PM, Luce JM, et al. Diagnostic utility of fibre-optic bronchoscopy in patients with Pneumocystis carinii pneumonia and AIDS. Am Rev Respir Dis 1983;128: 795-9. 